\title{
Mobile Robotic Surveying Performance for Planetary Surface Site Characterization
}

\author{
Edward Tunstel ${ }^{1}$, John M. Dolan ${ }^{2}$, Terrence Fong ${ }^{3}$, Debra Schreckenghost ${ }^{4}$ \\ ${ }^{1}$ Space Department, Johns Hopkins Univ. Applied Physics Laboratory, Laurel, MD 20723 USA, \\ edward.tunstel@jhuapl.edu \\ ${ }^{2}$ Robotics Institute, Carnegie Mellon University, 5000 Forbes Ave., Pittsburgh, PA 15213 USA, \\ jmd@cs.cmu.edu \\ ${ }^{3}$ Intelligent Robotics Group, NASA Ames Research Center, Moffett Field, CA 94035 USA, \\ terry.fong@nasa.gov \\ TRACLabs, Inc., Houston, TX 77058 USA, schreck@traclabs.com
}

\begin{abstract}
Robotic systems will perform mobile surveys for scientific and engineering purposes as part of future missions on lunar and planetary surfaces. With site characterization as a task objective various system configurations and surveying techniques are possible. This chapter describes several examples of mobile surveying approaches using local and remote sensing configurations. A geometric measure of area coverage performance is applied to each and relative performance in surveying a common area is characterized by expected performance trends. Performance metrics that solely express geometric aspects of the robotic task are limited in utility as decision aids to human mission operators. As such, the importance of enriching such metrics by incorporating additional attributes germane to surveying on planetary surfaces is highlighted. Examples of enriched metrics employed by recent NASA research work on human-supervised robotic surveying are provided.
\end{abstract}

Keywords: robotic surveying, planetary surface exploration, in-situ remote sensing, area coverage performance, site characterization, resource prospecting, human supervision, consolidated performance metrics, real-time performance metrics.

\section{Introduction}

On Earth, the exploration and settlement of uncharted territories requires prior prospecting and surveying for useful resources. The same is true for exploration and eventual human settlement of outposts on planet surfaces. Space agencies rely on precursor robotic missions to acquire the data and information necessary to un- 
derstand planetary surface regions and the feasibility of sending human explorers on future missions. Surveying refers to the systematic method or technique of making measurements essential for accurately determining the geo-spatial location of commodities of interest in a designated area. Prospecting refers to the methodical and qualitative physical search or exploration for the commodity. Intelligent robots will need to be equipped with effective techniques for performing these tasks in preparation for the return of astronauts to the Moon and exploration of planetary surfaces beyond.

Task-oriented algorithms that support systematic mobile surveys using science instruments are needed for planetary surface characterization on science missions. They are also needed for in-situ resource prospecting and mapping on robotic missions that serve as precursors to human exploration missions. Typical objectives of site surveys include sensor coverage of designated areas. Area coverage problems for mobile robotic survey systems commonly employ sensing devices requiring close proximity to or contact with the measured phenomenon. Examples of such "local sensing" devices include ground penetrating radar, metal detectors for humanitarian de-mining, fluorescence imagers for organic molecule detection, and various spectrometer types. Mobile robotic vehicles, or rovers, that carry survey systems comprised of local sensing devices must physically cover most, if not all, of the terrain in the designated survey area.

Remote sensor-based area coverage contrasts with these more common area coverage problems for mobile robotic surveys. Remote sensing instruments can acquire measurements at significant distances away from the measured phenomenon (e.g., based on radar or optical devices such as lasers). Measurements along a line-of-sight to detect airborne phenomena such as near-surface gas emissions, for example, account for coverage of terrain below that line-of-sight. This permits a two-dimensional search over terrain using discrete linear measurements from a distance (similar to scanning laser rangefinders).

Remote sensor-based methods are not applicable to all surveying tasks. For surveys in which they are not a better solution, they are often excellent complements to local sensor-based methods. That is, remote sensor-based surveys can serve as an efficient means to cover wide areas with the purpose of localizing smaller areas at which local sensor-based surveys of higher resolution are appropriate.

Mobility algorithms for surveying provide a means to systematically acquire measurements covering an area to be surveyed by transporting sensors and instruments to multiple locations and vantage points. Algorithms employing parallel-line transects or parallel swaths are commonly used to address robotic area coverage problems by single robots [1,2] and multiple robots [3] when using local sensing devices. Full and partial coverage planners have also been proposed for rovers that survey terrain using local sensing devices [4]. Random walk and chaotic coverage trajectories are also possible $[5,6]$ but less popular for systematic coverage tasks. Remote sensor-based survey approaches for rovers have recently 
been proposed for single- and two-rover systems performing measurements through the near-surface atmosphere [7,8].

This chapter examines several examples of both mobile survey types and applies a geometric measure of their area coverage performance. It further advocates the importance of additional attributes germane to surveying tasks for planetary surface exploration and presents representative examples from NASA-funded robotics research. The additional attributes are intended to enrich the effectiveness and relevance of basic geometric measures or support formulation of new metrics for intelligent/autonomous robotic survey systems in planetary surface domains.

\section{Local Sensor-Based Surveying}

Due to required proximity to measured phenomena and relatively small footprints of sensitivity, local sensing devices typically necessitate dense coverage of a designated survey region by the host mobile platform. As such, local sensor-based mobile surveys seek to acquire measurements that cumulatively cover all or most of the survey region. Associated survey sensors or instruments are typically mounted on a rover body or deployed on a rover-attached boom or manipulator arm. Rover mobility serves to transport the footprint of the survey instrument(s) over terrain along trajectories that fill the survey region. Fig. 1 depicts this scenario.

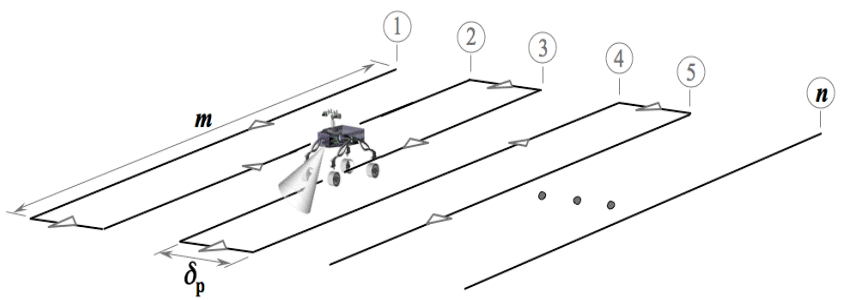

Fig. 1. Local sensor-based surveying along $n$ parallel transects of length $m$.

Among alternative survey trajectories for the mobile platform, parallel transects, spirals, and random walks have been proposed. Parallel transects are most commonly applied for coverage tasks although a spiral coverage approach was proposed for mobile mining of regolith on the lunar surface as part of a so-called spiral mining system [9]. Recent field tests, focused on planetary surface site characterization, used a rover to perform surveys using a ground penetrating radar (GPR) along parallel transects covering a $700 \mathrm{~m}$ x $700 \mathrm{~m}$ survey region [10]. This instrument was used to map the subsurface structure at the site. A total traverse distance of $20.5 \mathrm{~km}$ was sufficient to cover the survey region using densely spaced parallel transects (with no overlap of the GPR sensor footprint on adjacent tran- 
sects) [10]. North-south and east-west transects were planned and executed. Parallel transect trajectories were also suggested for systematic resource prospecting of wide areas on the lunar surface [11].

Basic geometric measures are often used to measure area coverage performance of such local sensor-based survey algorithms. Examples include measures of distance traveled, rover drive- and run-time [10], and percent of total area covered [2]. Variants of the latter have been proposed based on distribution of measurement samples within the cells of a tessellated grid representation of the survey region [4]. Here, we apply a basic geometric measure comprising a combination of such attributes. It is referred to as the quality of performance, $Q o P$, defined as a ratio of area covered to distance traveled [12]. Applying this metric to the recent field test result mentioned above would yield a QoP of 24 based on the survey region area and total traverse distance (note that the north-south and east-west transects performed in that field test covered the survey region twice, effectively). In theory, an optimal value for this metric might be associated with the minimum distance traversed while acquiring non-overlapping survey sensor measurements; although in practice, it may depend on a variety of sensor, system, and task characteristics. In general, the QoP for a local sensor-based survey along parallel transects (Fig. 1) is computed as

$$
Q_{p}=\frac{m(n-1) \delta_{p}}{\left[m n+(n-1) \delta_{p}\right]}
$$

where $m$ is the transect length, $n$ is the number of transects traversed, and $\delta_{\mathrm{p}}$ is the separation distance between adjacent transects and is assumed here to be comparable to the survey sensor footprint. The numerator and denominator of Eq. (1) respectively represent the area surveyed and total distance traversed during the survey.

In the next section we discuss mobile surveying using in-situ remote sensing and apply the same metric to area coverage performance of several types of remote sensor-based survey trajectories.

\section{Remote Sensor-Based Surveying}

Mobile remote sensor-based surveys can be performed by measurement systems whose components are separated by a distance across terrain. Such systems are comprised of an active/passive instrument component on one end and a passive/active component on the other end. One end could be stationary while the other is mobile (fixed-mobile) or both ends could be mobile (mobile-mobile). Both are considered here beginning with a fixed-mobile configuration, which is suitable for single-site surveys (unless the fixed component is also transportable to other sites). 


\subsection{Single-Site Remote Sensing Surveys}

Consider a fixed-mobile configuration comprised of an active rover-mounted instrument, a passive receiver or retroreflector at a fixed location a distance away, a rover pan-tilt unit to point the instrument at the passive component for measurements, and the rover itself to move the instrument spatially over terrain. The passive component would remain stationary at a position central to a designated survey region. This configuration is similar to those of Total Station systems commonly used by civil engineers for land surveys and comprised of a theodolite on one end and stationary $360^{\circ}$ retroreflector on the other. Like a civil engineer, a rover using such a survey system can acquire measurements from any radial direction when the fixed component is within line-of-sight and measurement range.

For mobile surveys, measurements are coordinated with rover mobility to survey terrain via a series of measurements across a distance $d$, which varies with rover position relative to the fixed component (Fig. 2). Such mobile robotic systems are under development for planetary surface surveying to achieve optical measurements at maximum distances of hundreds of meters [13]. The long-range measurement capability coupled with rover mobility enables wide-area surveys.

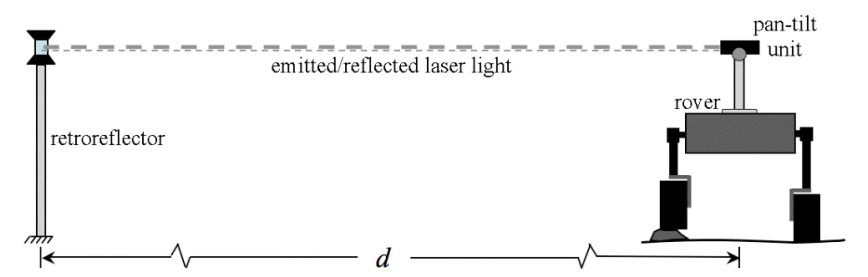

Fig. 2. Example of a distributed fixed-mobile measurement configuration.

Concentric circular or spiral trajectories are compatible with distributed fixedmobile configurations for remote sensor-based surveying. A designated survey region with a fixed instrument component at its center can be covered by traversing concentric circular trajectories as follows. The location of the fixed component is known and considered to be the origin of an inertial coordinate system in which the survey region and task is defined. Rover pose during surveys is estimated relative to this coordinate system. Beginning at a designated radial distance from the fixed component, the rover moves in arc-increments stopping periodically on the trajectory to acquire measurements. We refer to these measurement locations as m-nodes. Measurements along a line-of-sight between the rover-mounted active instrument and the fixed component account for 2-D coverage of terrain below the line-of-sight. Such measurement techniques are used on Earth with laser-based spectrometers to probe for and detect gas emissions during environmental site surveys [14], and they are being developed for the same fundamental use on Mars [13]. An accumulation of such linear measurements from discrete radial locations and distances achieves survey region coverage. 
The following four parameters are used to configure a concentric circular trajectory covering a given survey region (Fig. 3): innermost circle radius, $\rho_{l}$; radial distance, $\delta_{\mathrm{c}}$, between circumferences of consecutive circles; arc length, $s$, between consecutive m-nodes on a circle; and positive integer, $n$, designating the $n^{\text {th }}$ or outermost circle including the survey region. The algorithm assumes that the rover is already within the survey region and that the fixed component is within line-ofsight from the rover [7]. If it is not, then no measurement is made. The survey completes when the $n^{\text {th }}$ circular trajectory is followed.

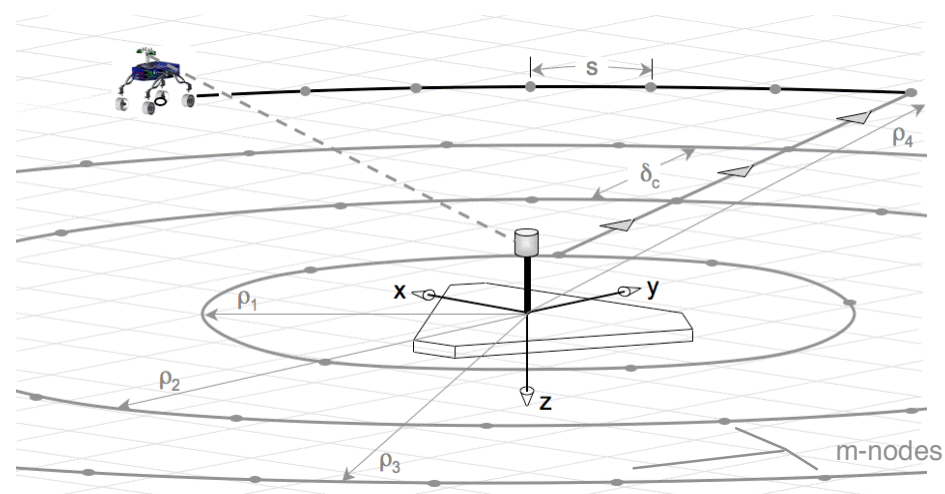

Fig. 3. Concentric circular remote sensing survey and parameters.

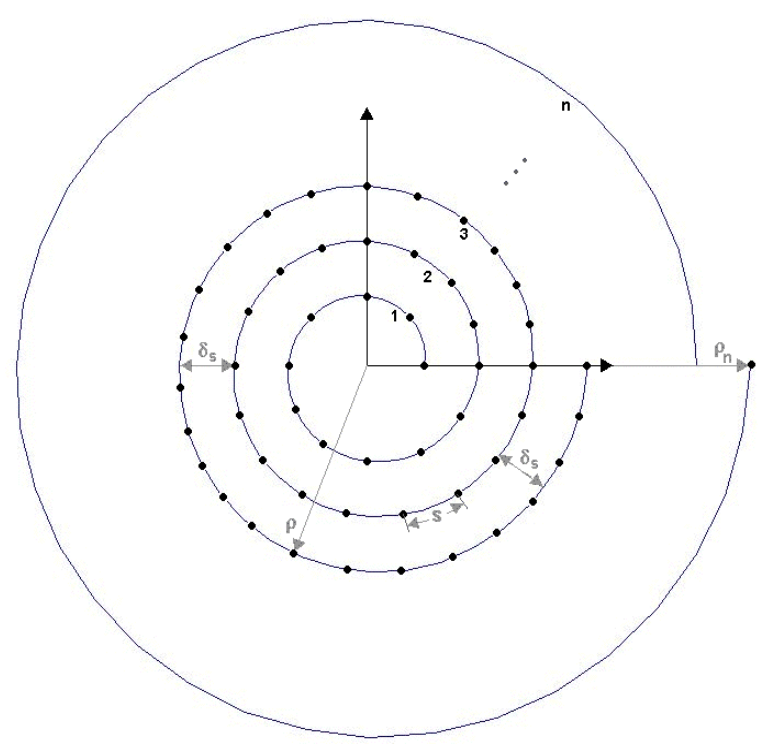

Fig. 4. Overhead view of spiral remote sensing survey and parameters. 
A fixed-mobile configuration performs a spiral survey in a similar manner, differing only in that the rover drives in arcs along a trajectory of continuously increasing radius and needs no specific maneuvers to transition between successive spiral branches at larger radii [7]. Fig. 4 illustrates the spiral survey trajectory, which is parametrically similar to a circular survey.

Both surveys are configured in a flexible manner to achieve desired degrees of measurement and area coverage resolution using the four parameters $(\rho, \delta, s, n)$. The surveys are primarily constrained by rover kinematic limitations, instrument effective minimum and maximum ranges, and terrain topography in the survey region whether executed radially inward or outward.

\subsection{Single-Site Remote Survey Performance}

Related research on distributed surveying [12] introduced the quality of performance metric defined earlier. We also apply this metric here as a basis for comparing expected performance of the concentric circular and spiral trajectories for distributed surveying.

The area of a survey region covered by either a concentric circular or linear spiral trajectory is equal to or roughly the same as $A=\pi \rho_{n}{ }^{2}$, where $\rho_{n}$ is the radius of the outermost circle or spiral branch. Areas within the survey region that are occupied by the stationary instrument component (at the origin of the survey coordinate system) and non-traversable obstacles are neglected. The total traverse distance $D_{c}$ required for a concentric circular survey is the sum of distances traveled on each circumference and the radial separation distances, $\delta_{c}$, between them:

$$
D_{c}=2 \pi\left(\sum_{i=1}^{n} \rho_{i}\right)+(n-1) \delta_{c}
$$

yielding the following QoP,

$$
Q_{c}=\frac{\pi \rho_{n}^{2}}{2 \pi\left(\sum_{i=1}^{n} \rho_{i}\right)+(n-1) \delta_{c}} .
$$

For each linear spiral branch traversed (every $\theta=2 \pi$ radians), the spiral radius $\rho$ increases by $\delta_{\mathrm{s}}$ (Fig. 4), i.e., $\rho=\left(\delta_{\mathrm{s}} / 2 \pi\right) \theta$. It can be shown [15] that the total traverse distance $D_{s}$ required for a linear spiral trajectory is then expressed as

$$
D_{s}=\frac{\delta_{s}}{4 \pi} \theta_{n}^{2}
$$


where $\theta_{n}$ is the maximum spiral angle reached. The resulting QoP is then

$$
Q_{s}=\frac{4 \pi^{2} \rho_{n}^{2}}{\delta_{s} \theta_{n}^{2}}
$$

Based on the QoP metric the two fixed-mobile configurations for remote sensor-based surveying can be compared. With roughly the same survey region area, their QoPs are distinguished by distance traveled. If the spiral begins and ends as shown in Fig. 4, then $\theta_{n}=2 n \pi$, and $D_{s}=\delta_{\mathrm{s}} \pi n^{2}$. For closest equivalence between the two trajectories, let the first circle radius be equal to the initial spiral radius, $\rho_{l}$, and let $\rho_{1}=\delta_{\mathrm{c}}=\delta_{\mathrm{s}}$. Under these conditions, $\rho_{2}=\rho_{1}+\delta_{\mathrm{c}}=2 \delta_{\mathrm{c}}, \rho_{3}=3 \delta_{\mathrm{c}}, \rho_{4}=4 \delta_{\mathrm{c}}$, and so on. The summation term in Eq. (2) then becomes a function of an arithmetic series and simplifies as follows.

$$
\begin{aligned}
\sum_{i=1}^{n} \rho_{i} & =\delta_{c}+2 \delta_{c}+2 \delta_{c}+\ldots+n \delta_{c} \\
& =\delta_{c}(1+2+3+\ldots+n) \\
& =\delta_{c}\left[\frac{1}{2} n(n+1)\right]
\end{aligned}
$$

Using this result in Eq. (2), we have

$$
\begin{aligned}
D_{c}= & 2 \pi \delta_{c}\left[\frac{1}{2} n(n+1)\right]+(n-1) \delta_{c} \\
= & \delta_{c}\left[\pi n^{2}+(\pi+1) n-1\right] .
\end{aligned}
$$

Therefore, $D_{c}>D_{s}$, independent of an equivalent separation distance. A rover executing a concentric circular survey of $n$ circles would need to traverse over $(4 n-1) \delta_{c}$ meters more to cover the same area as it could with a spiral trajectory of $n$ branches. As an example, to traverse a survey trajectory of $n=3$ concentric circles separated by $\delta_{\mathrm{c}}=10 \mathrm{~m}$, a rover would drive a linear distance of $397 \mathrm{~m}$; to survey a roughly equivalent area using a spiral trajectory of $n=3$ branches separated by $\delta_{\mathrm{s}}=10 \mathrm{~m}$ it would drive a linear distance of $283 \mathrm{~m}$, or $29 \%$ less.

\subsection{Multiple-Site Remote Sensing Surveys}

An example of a mobile-mobile, or tandem, survey system configuration is illustrated in Fig. 5. Both rovers could carry the active and passive components of the distributed survey instrument (e.g., for redundancy), or each rover could carry the 
companion component to the other's payload. Such survey configurations are suitable for multiple-site surveys due to the mobility of both platforms. The same dual mobility enables this tandem configuration to perform a number of approaches to remote sensor-based mobile surveying including, as special cases, the approaches described above for single-site surveys.

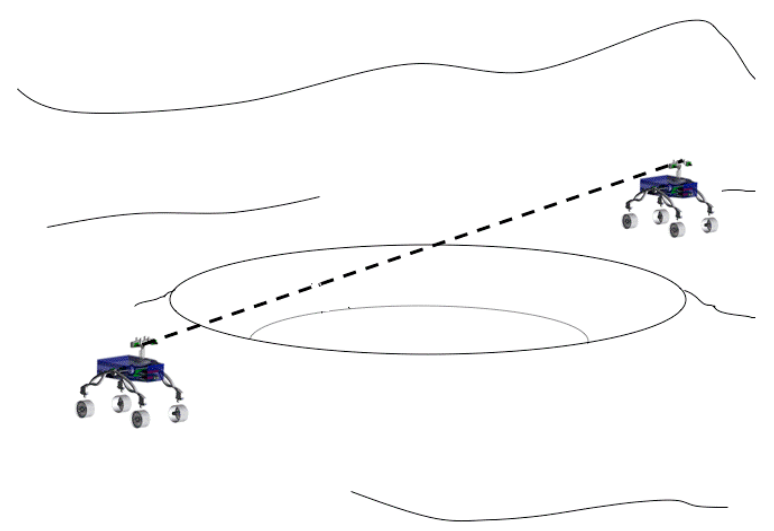

Fig. 5. Tandem distributed remote sensing configuration.

\subsection{Multi-Site Remote Survey Performance}

A tandem-robot system was proposed in $[8,12]$ in which one robot carried an active instrument and the other carried the instrument's passive receiver. The robots would cooperatively perform remote sensor-based surveys using either parallelswath or circular patterns where the width of a swath is the separation distance between the robots. The QoP metric was applied to compare several variants of these survey patterns including those illustrated below in Fig. 6. These survey approaches are referred to here as tandem-parallel and tandem-circular, and the QoPs for each are:

$$
\begin{gathered}
Q_{t p}=\frac{m n \delta_{t p}}{\left[2 m n+2(n-1) \delta_{t p}\right]} \\
Q_{t c}=\frac{\pi\left(n \delta_{t c} / 2\right)^{2}}{\frac{\delta_{t c}}{2}\left(\pi n^{2}+2 n-4\right)}
\end{gathered}
$$


where $m$ is the length of a surveyed parallel swath (see Fig. 6), $\delta_{\mathrm{tp}}$ and $\delta_{\mathrm{tc}}$ are the robot separation/measurement distances during a survey, and $n$ (in both equations) is the total number of swaths surveyed [12]. The denominators of Eqs. (8) and (9) express the total distances, $D_{t p}$ and $D_{t c}$, traversed by both robots for the respective tandem survey types.
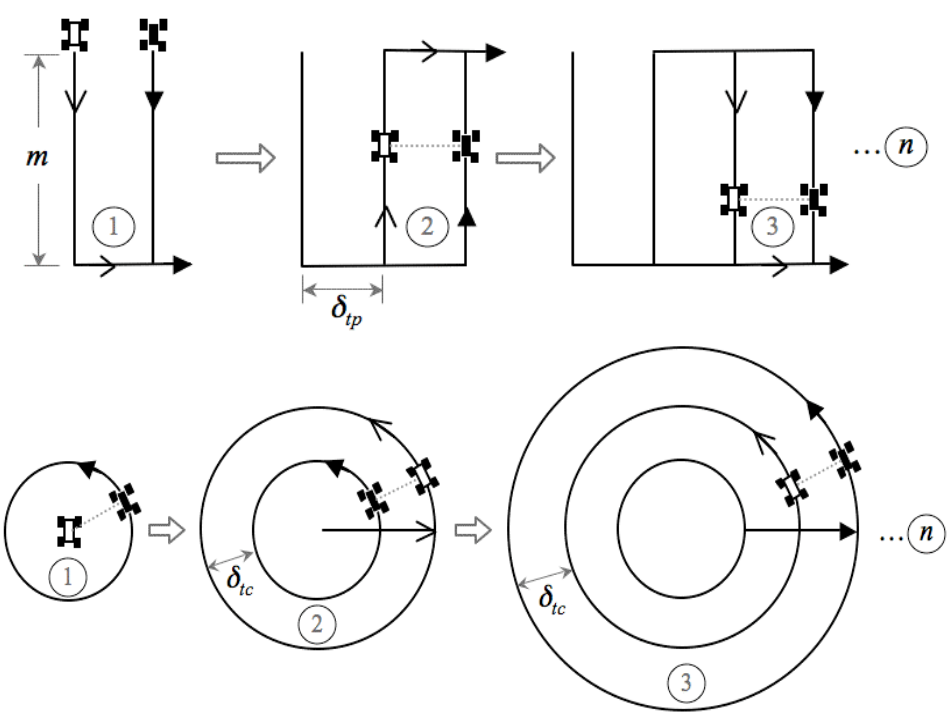

Fig. 6. Tandem parallel and circular remote sensing survey trajectories.

\section{Characteristic Performance of Mobile Surveys}

The use of a common performance metric to comparatively rank a set of options provides a valuable basis or choosing the best option for a given task. However, direct one-to-one comparisons of the mobile surveying approaches discussed above are not straightforward given their respective differences in survey system configuration and survey trajectories. In fact, they can be prescribed for considerably different types of survey tasks in practice. Nonetheless, somewhat common grounds for comparison would provide a general sense for the relative performance of each approach. As a useful compromise a characteristic comparison is made here, still using a common metric, but based on assumptions that serve to equalize differences across the set of options. We use the QoP as the common metric here but any of a variety of metrics could be used instead.

Consider a mobile survey of a designated region with common area, $A$, and performed by each of the local and remote sensor-based methods discussed above. Recall that the metric for each survey type is the total area covered divided by the 
total distance traversed, $D$, during the survey $(\mathrm{QoP}=A / D)$. Since $A$ is considered to be a common survey region size, differences in the QoP are functions of differences in $D$ only. Further assume that each survey is designed for a comparable area coverage resolution; this would call for equal values of the parameters $\delta_{\mathrm{p}}, \delta_{\mathrm{c}}$, $\delta_{\mathrm{s}}, \delta_{\mathrm{tp}}$, and $\delta_{\mathrm{tc}}$. For the respective QoPs given by Eqs. (1), (3), (5), (8), and (9), the distance traveled is a function of the area coverage resolution. For simplicity, we assume a value of unity as the common value for the respective resolution parameters. With this assumption the expression of distance traversed for each QoP becomes a function of parameter $n$ only, with the exception of $Q_{\mathrm{p}}$ and $Q_{\mathrm{tp}}$, i.e., the local parallel and tandem-parallel survey metrics in Eqs. (1) and (8). If we assume a square survey region, then the length of a transect for a local parallel survey becomes $m=(n-1) \delta_{\mathrm{p}}=(n-1)$, since $\delta_{\mathrm{p}}$ is set to unity. Similarly, the length of a swath for a remote tandem-parallel survey becomes $m=n \delta_{\mathrm{tp}}=n$, since $\delta_{\mathrm{tp}}$ is set to unity. With all traverse distances as functions solely of the $n$-parameter for each survey type, the following characteristic expressions, $D(n)$, apply

$$
\begin{aligned}
& D_{p}(n)=n^{2}-1 \\
& D_{c}(n)=\pi n^{2}+(\pi+1) n-1 \\
& D_{s}(n)=\pi n^{2} \\
& D_{t p}(n)=2 n^{2}+2(n-1) \\
& D_{t c}(n)=\frac{1}{2} \pi n^{2}+n-2 .
\end{aligned}
$$

Note that Eqs. (10)-(14) are only characteristic of the respective distances traversed due to the different meanings for $n$ for each survey type, i.e., the number of parallel transects, concentric circles, spiral branches, and parallel swaths. For this discussion we will generally refer to $n$ as the number of survey passes. Fig. 7 shows the characteristic trends of traverse distances required by each mobile survey configuration to cover the same area with comparable coverage resolution. The characteristic trends are shown for up to 10 survey passes only. They effectively reveal the sensitivity of the QoP metric to $D$, based on assumptions made above.

As the number of survey passes increases, the characteristic distances for the circular and spiral remote sensor-based surveys increase at the fastest and roughly the same rate. Circular remote-sensing surveys require the longest characteristic traverse distances. The QoPs for these fixed-mobile surveys would be expected to decrease most rapidly with high numbers of survey passes, while higher QoPs would be expected for spiral surveys with low numbers of passes. For the tandem configurations, parallel circular surveys would be expected to perform better than parallel swath surveys, which would require characteristically longer traverse distances. The QoPs for tandem approaches would be expected to be higher than those of fixed-mobile approaches for the same survey region and coverage resolution. This reflects the advantage of using more than one rover for distributed re- 
mote sensing survey tasks [8]. The popular parallel transect survey approach for single rovers with local sensing devices has the slowest trend of increasing distance as the number of survey passes increases. Thus, its QoP would be expected to be high and least impacted by $n$, relative to the other approaches, for surveying the same area at a comparable coverage resolution.

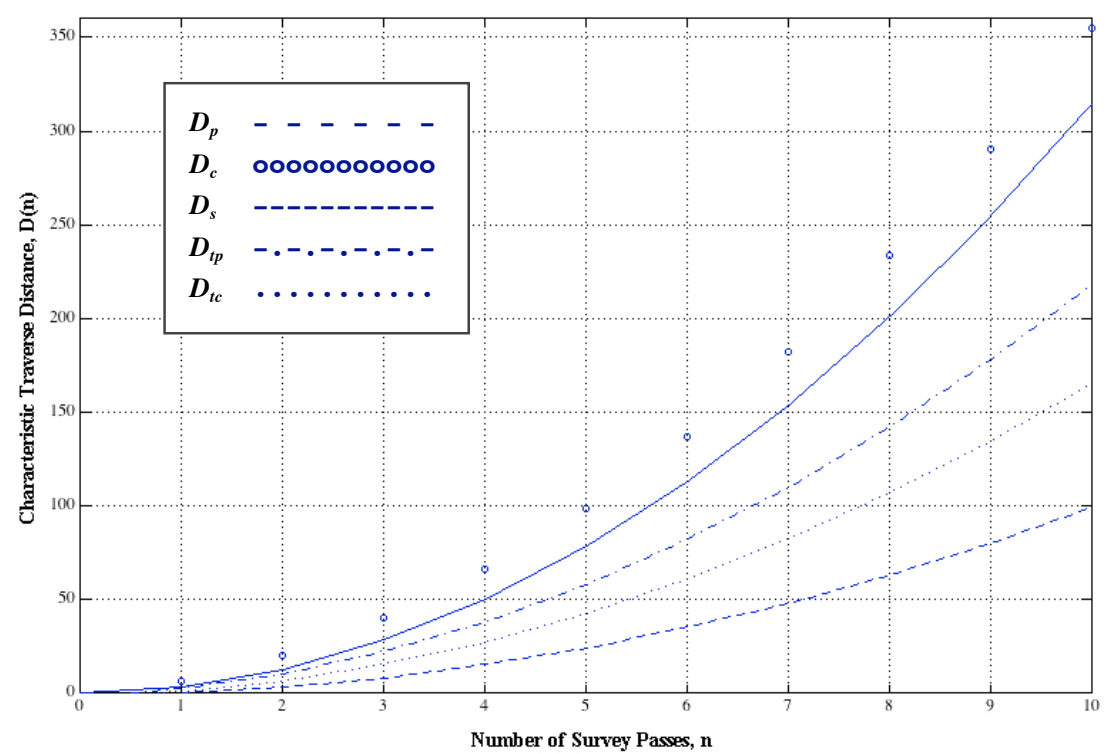

Fig. 7. Characteristic traverse distance trends for mobile survey configurations as a function of the number of survey passes.

\section{Enriching Metrics for Surveys on Planetary Surfaces}

Thus far we have considered evaluation of coverage performance for surveys based on simple geometric measures of area and distance. Other important measures should be considered to enrich performance evaluation in the domain of planetary robotic systems and otherwise resource-constrained field robot systems. In fact, the selection of a candidate survey trajectory type for a given survey region can be based on metrics that provide some measure of relative resource usage during trajectory following (e.g., time, power, survey data storage). Resources can be included in consideration of distance so that, from a resource usage point of view, the highest QoP for a given mobile surveying system and area to be covered would be associated with the shortest traverse distance required for that system to cover the area. In that case, the most practical choice among possible survey tra- 
jectories may be the trajectory that requires the least resources to traverse the shortest distance resulting in area coverage by the survey sensor(s). Selection of a survey type can also be based on any known physical constraints about the survey region such as terrain topography or the size and distribution of phenomena to be measured (e.g., as derived from earlier orbital reconnaissance or prospecting efforts). Past work provides guidance in this direction. For example, maximization of incident solar energy on rover solar arrays has been considered as a determinant for selection among several survey trajectory types [16]. Another study applied an energy efficiency metric, defined as a ratio of area covered to energy consumed, to evaluate parallel line, circular spiral, and square spiral trajectories [15]. Other attributes can be adopted that are germane to surveying for planetary surface exploration but proposed in the context of mobility/navigation or task performance. These include terrain traversability measures such as obstacle abundance [17, 18] and number or effort of rovers and human operators involved [11].

While identifying domain specific attributes that would enrich the relevance of existing geometric measures is helpful, the manner in which they would be incorporated into a metric formulation is also worth considering. Metric formulations that are computationally complicated or multi-dimensional can be difficult to apply, hard to interpret, or both. In the domain of planetary site characterization, a number of system, mission, and/or environmental constraints will affect the performance of mobile surveying tasks. Performance metrics based solely on geometric aspects of the task do not capture other important performance impacts of the task, and therefore are particularly limited as decision aids. Human mission operators or task supervisors will be better equipped to select appropriate survey methods when using metrics that account for a broader range of performance impacts that include system resources, terrain information or task constraints in addition to geometric measures like the QoP.

As one example of how a resource attribute can change and influence the effectiveness of a basic metric consider the following. In [15], energy consumed by robot wheel motors was considered based on an empirically derived model of DC motors. Differences in energy efficiency were attributed in part to the required amount of turns the robot must execute to follow the search pattern. Depending on how a rover mobility system is kinematically constrained to execute a traverse, many turns-in-place may be necessary throughout a survey. Such maneuvers are not captured by a metric like the QoP and unless energy consumption (at least) is considered, the overall performance of a survey could be obscured. The study considered energy consumed during accelerations in addition to during turns, which led to a conclusion that circular spiral surveys were most efficient for larger survey areas while parallel line scans were most efficient for small areas [15]. This conclusion is based on the fact that the robot continuously moves without stopping and turning when executing spiral trajectories, thus consuming less energy over longer distances required for spiral surveys. Finally, consider that while distance traversed and energy consumed are correlated in most cases, if terrain traversability is ignored then a mobile surveying metric will not capture the distance or en- 
ergy impacts of surveying a rough and rocky terrain cluttered with obstacles. Due to such considerations, we advocate for enrichment of metrics for mobile surveying tasks to improve their utility as decision aids for actual mission operations.

\subsection{Consolidated Metric for Human-Supervised Robotic Prospecting}

NASA-funded research on human-supervised autonomy and execution of robotic tasks in the context of lunar surface operations has focused on approaches for both scientific and engineering surveying. A Robot Supervision Architecture (RSA) for human tele-supervision of a fleet of mobile robots was presented in [11]. The RSA was focused on the scientific task of autonomously searching an area for in-situ resources/minerals to demonstrate human-robot interactions during tele-supervised prospecting and to validate prospecting performance. Fig. 8 depicts a typical deployment scenario: one or more human astronauts supervise multiple robots from a climate-controlled habitat ("Base"). In the depicted example, four robots are dispatched from the Base and prospect at fixed intervals using a parallel transect path as described in Section 2. Surface sensing and other prospecting science would be performed at discrete prospecting sites.

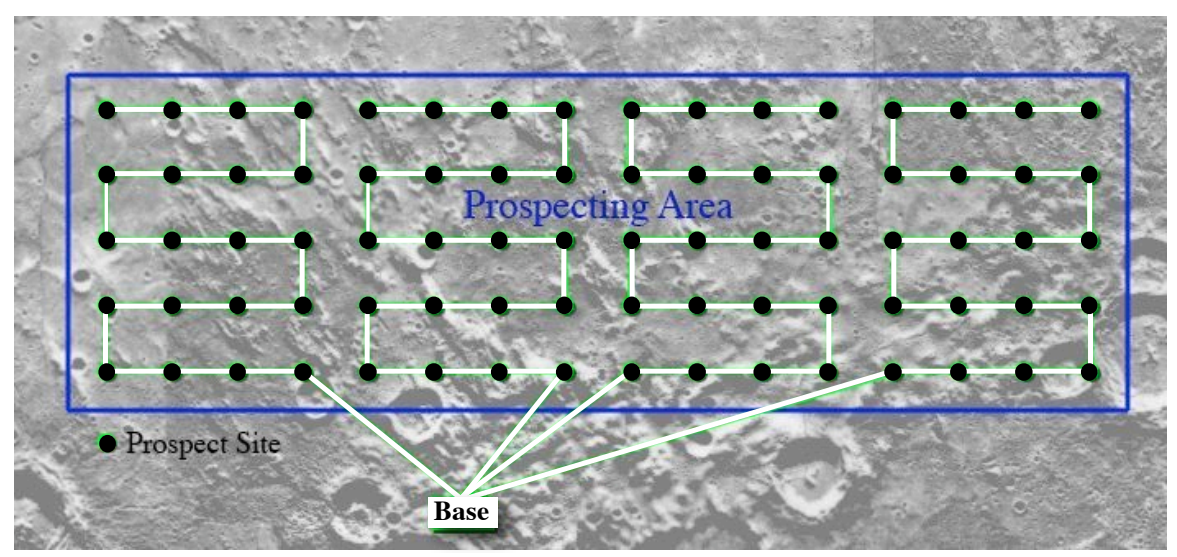

Fig. 8. Robot paths within prospecting area.

Many factors potentially relate to the performance of a human-supervised multi-robot system of this sort. Instead of using multiple individual and independent metrics, such as those described in [19], we propose a consolidated performance metric based on the following notions: 1) greater area, accuracy, terrain difficulty, and safety of prospecting coverage mean increased performance; 2) greater effort and time required mean decreased performance. The consolidated performance metric is thus expressed as follows [11]: 


$$
P=\frac{A C T S}{\left(R / w+H_{E}\right) t}
$$

where:

$P$ : performance in units of (area accurately and safely prospected)/(effort-time) $A$ : area covered

$C$ : prospecting accuracy; $C=1$ corresponds to the highest possible accuracy and $C$ $=0$ corresponds to the lowest possible accuracy

$T$ : terrain difficulty factor ( $T \geq 1)$ with $T=1$ corresponding to the easiest terrain (a flat surface without obstacles)

$S$ : safety factor $(S \geq 1)$ with $S=1$ corresponding to the least safe task performance, i.e., one requiring $100 \%$ extra-vehicular activity (EVA) for the astronauts

$R$ : number of robotic rovers (integer)

$H_{E}$ : human effort defined as the degree of human task intervention in full-time equivalents ( $0 \leq H_{E} \leq H$; $H$ is the integer number of humans); e.g., if one human intervenes $30 \mathrm{~min}$. during a 1-hr. task, $H_{E}=(30 / 60)=0.5$; if three humans intervene 15, 30, and $45 \mathrm{~min}$. respectively during a 1-hr. task, $H_{E}=(15 / 60)+$ $(30 / 60)+(45 / 60)=1.5$

$w$ : factor allowing commensurability of human and rover time by giving the relative value of the former to the latter; e.g., $w=4$ sets human time to be four times as valuable as rover time

$R / w+H_{E}$ : combined human-rover effort

$t=$ time required to cover $A$.

Note that for the purposes of the consolidated metric, $P$, the type of prospecting path used is of little relevance (except to the extent that it impacts $t$ ); different paths executed in comparable time periods would yield similar performance values. Of higher relevance, however, are the individual measures of prospecting accuracy, terrain difficulty, and safety included in the consolidated metric. These measures are important factors in the context of a planetary surface mission and further described as follows:

- Prospecting accuracy: This quantifies how accurately the system can identify regions of geological interest in the covered area. If the system covers a large area with little human effort, but fails to identify any of the existing regions of interest, its overall performance should be zero. This is reflected in the factor $C=(1-E)$ in the numerator of the Eq. (15). This factor ranges between 0 and 1 , where 0 corresponds to all directly observed regions being misestimated with the highest possible error (hence $E=1$ ), and 1 corresponds to all directly observed regions being correctly identified (hence $E=0$ ). $E$ is a normalized error metric over regions that were directly measured by the rovers.

- Terrain difficulty: This is reflected primarily or exclusively in a reduced average speed of the rovers. One could, for example, take as inputs the average obstacle size and density, the average terrain slope, and the average terrain trac- 
tion (and maybe other inputs) and calculate a "terrain difficulty factor" $T$ whereby the average rover speed is decreased with respect to a flat surface without obstacles, for which $T=1$. All other factors being equal, a higher terrain difficulty factor $T$ gives a higher performance, as reflected by its presence in the numerator of Eq. (15).

- Safety: A reasonable measure of safety is related to the percentage of EVA time the astronauts spend during the task. Let us define $V=(1-\%$ EVA time $)$, so that $V=1$ corresponds to no EVA time, and $V=0$ corresponds to all time being EVA time. Safety is difficult to make commensurable with performance as defined above, but a possibility is to define $S=(1+(\beta-1) V)$, in which case $V=$ 0 corresponds to $S=1$, or baseline minimally safe performance, and $V=1$ corresponds to $S=\beta$, or the safest possible performance. The subjective factor $\beta$ must be provided by human judgment and represents how much more valuable safe performance is than unsafe performance. If $\beta=4$, for example, then full safety with all other factors being equal results in a performance 4 times better than that achieved with minimal (maximum-EVA) safety.

A given performance target, $P_{\text {target }}$, calculated using Eq. (15) can be met in a variety of ways by a human-supervised multi-robot system. Such flexibility increases the utility of the metric as a decision aid to mission operators. As an illustration, consider a $100 \mathrm{~m} \times 100 \mathrm{~m}$ prospecting area, $A$, and assume maximum prospecting accuracy with minimum safety and terrain difficulty so that $C=T=S$ $=1$. As a subjective choice for this hypothetical prospecting task, let the number of humans controlling be $H=2$, and let human time be considered 5 times as valuable as rover time, i.e., $w=5$. The performance target for this task becomes $P_{\text {target }}=10 \mathrm{~km}^{2} /($ effort-hour $)=A /\left[\left(R / 5+H_{E}\right) t\right]$, which can then be achieved under the following scenarios for various amounts of area coverage time and human and robot effort:

1. $H_{E}=1, R=0, t=1$ hour: One human does the task alone in an hour.

2. $H_{E}=2, R=0, t=1 / 2$ hour: Two humans do the task with no rovers in $1 / 2$ hour.

3. $H_{E}=0, R=5, t=1$ hour: Five rovers do the task without human help in an hour.

4. $H_{E}=0, R=10, t=1 / 2$ hour: Ten rovers do the task without human help in a half-hour.

5. $H_{E}=1 / 5, R=4, t=1$ hour: Four rovers do the task with 12 minutes $(1 / 5 * 1$ hour) of human supervision in an hour.

6. $H_{E}=6 / 5, R=4, t=1 / 2$ hour: Four rovers do the task with 36 minutes $(6 / 5 * 1 / 2$ hour) of human supervision in $1 / 2$ hour or 30 minutes (both humans involved).

7. $H_{E}=6 / 5, R=9, t=1 / 3$ hour: Nine rovers do the task with 24 minutes $(6 / 5 * 1 / 3$ hour) of human supervision in $1 / 3$ hour or 20 minutes (both humans involved). 
Scenarios 1 and 2 together and 3 and 4 together show that doubling human effort or rover effort halves the time required for equivalent performance. Scenarios 1 and 3 together and 2 and 4 together show that one human doing the task is equivalent in performance to five rovers doing the task. Scenarios 3 and 5 show that, for the same time required, the removal of one rover's effort requires the provision of only $1 / 5$ of a human's effort. Scenarios 5 and 6 show that the addition of one human's effort with no increase in rovers halves the time, whereas scenarios 5 and 7 show that the addition of one human's effort along with five rovers' effort reduces the time by a factor of three.

\subsection{Metrics for Real-time Assessment of Robot Performance}

Beyond assessing performance in a purely analytic manner (i.e., for a priori planning or post-mortem analysis), we believe that it is also important to develop metrics that can be used during survey operations. In particular, we anticipate that future lunar surface surveys will be conducted with robots that are remotely supervised initially by Earth-based operators and later by operators situated in lunar habitats as discussed above. Ground control teams (including scientists and engineers) or operators at telesupervisor workstations in lunar habitats will need to monitor robot performance in order to dynamically adjust robot plans.

Some of our work $[19,20]$ has addressed defining and computing performance metrics in real-time. Our approach is to monitor data streams from robots, compute performance metrics in-line, and provide Web-based displays of these metrics. Our displays provide summary views of current performance as well as timeline plots, which are useful for spotting trends and key events. We have identified three categories of metrics that are particularly germane to real-time monitoring:

- Mission: metrics that describe the robot's contribution to the mission. For example, "Work Efficiency Index" [21] describes the ratio of productive time on task to overhead time. When WEI exceeds 1.0, the robot is spending more time accomplishing mission objectives, i.e., more productive.

- Task: metrics that describe the rover's performance with respect to the task (e.g., survey) plan. For example, "Percentage Distance Complete" summarizes the percentage of the planned distance that has been traveled by the rover.

- Robot: metrics that describe the robot's health and status. For remotely operated, or supervised, robots, two useful metrics inspired by reliability techniques are "Mean Time to Intervene" (MTTI) and "Mean Time Between Interventions" (MTBI) [22]. 


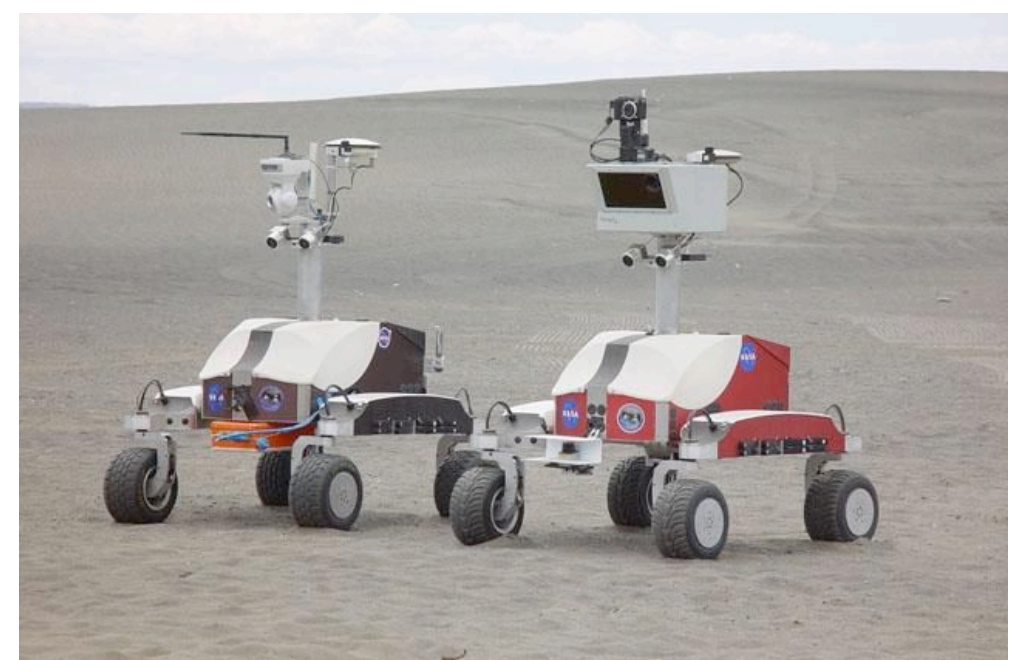

Fig. 9. NASA Ames K10 planetary rovers at Moses Lake Sand Dunes.

We evaluated our approach for real-time performance assessment during a field test at Moses Lake Sand Dunes (in Moses Lake, WA USA) in June 2008 [23]. In this test, we used two NASA Ames K10 planetary rovers (Fig. 9) to perform scouting and survey operations. The K10's were equipped with $3 \mathrm{D}$ scanning lidar, panoramic and high-resolution terrain imagers, and ground-penetrating radar. We simulated remote lunar science operations with a ground control team located at the NASA Johnson Space Center (Houston, TX USA).
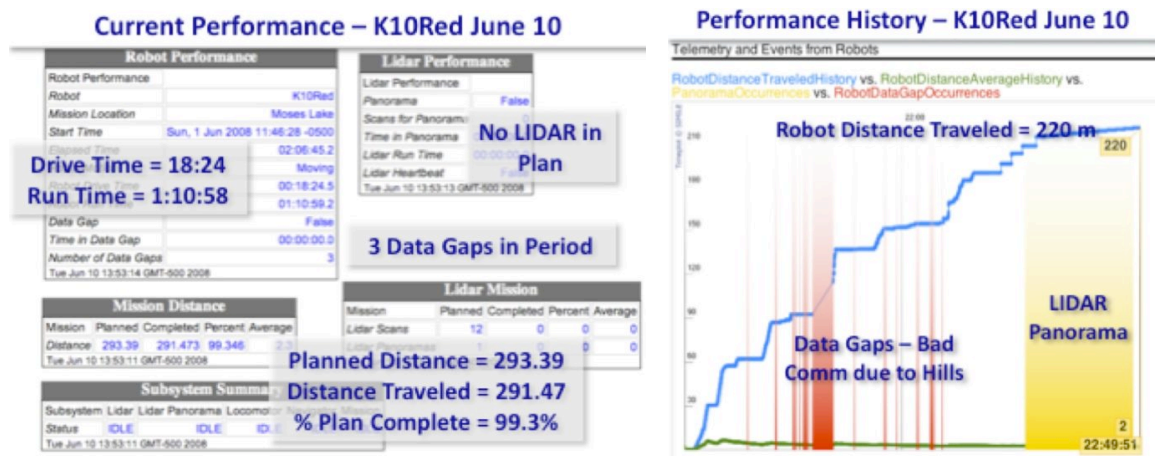

Fig. 10. Real-time robot performance displays. Left, "dashboard" summary; right: timeline plot.

Fig. 10 shows two real-time performance displays from the Moses Lake test. The "dashboard" provided ground control with summary metrics including robot task performance (drive time, \% distance complete, etc.), instrument performance (run time, completed measurements, etc.) and communication quality (e.g., data 
gaps). The timeline display provided operators with a time-based plot of the history of performance values. A detailed description these displays and how metrics were computed, interpreted and used is presented in [20].

\subsubsection{Considerations for Real-time Robot Performance Assessment}

Understanding the intent of rover activities provides an important basis for grounding the computation and interpretation of performance metrics. In particular, we assert that metrics, whether used for real-time monitoring or post-mortem analysis, can only be interpreted in context, i.e., with respect to expectations of performance for a particular task, activity plan, robot mode of operation, etc.

For example, the computation of metrics during autonomous operations should be distinguishable from metrics during direct teleoperation because expected performance can be quite different in these different control modes. In addition, the computation of metrics during anomalous operations should be separable from nominal operations. This is because a robot can be considered to perform "well" if it recovers quickly from a contingency, while at the same time performing poorly on nominal mission objectives.

For operations that involve human supervision, we also contend that performance assessment should consider only time periods when the rover is intended to be performing tasks [20]. Thus, we define a concept called a "duty period" that corresponds to a contiguous interval of operations, such as from rover startup until a planned ground-control break period. This helps reduce bias caused by idle time in the computation of metrics.

\section{Summary and Conclusions}

Science and engineering surveys will need to be conducted by mobile robotic systems to characterize sites on planetary surface missions. Various system configurations and surveying techniques are possible and performance metrics provide a basis for evaluating the options. Geometric metrics are a useful starting point for assessing expected survey performance in terms of coverage. However, other measures, such as system resources, terrain information, or mission-related task constraints should be considered in order to make a complete evaluation. While this chapter has focused on survey by wheeled rovers it should be noted that the discussion applies equally well to walking or flying robots.

This chapter illustrates how the QoP metric can provide a basis for performance comparison of different types of mobile survey trajectories, and additional metrics that can supplement such coverage metrics are discussed. When computing these metrics for a rover in the field or on a mission, however, it is important to consider how the environment, the equipment, and the mission affect the inter- 
pretation of computed values. For example, terrain traversability can impact both the distance traveled and the energy expended by the rover.

Through our work, we have found that expectations about nominal performance can be strongly influenced by environmental conditions. In addition, nominal performance can be degraded over the course of multiple missions due to component wear or systemic problems. In general, the limits imposed by component use and subsystem problems define the expectations about robot degraded mode performance. Robot operations can be further constrained by flight rules, i.e. mission-imposed constraints on operation. Mission constraints define safe rover behaviors. For robotic survey, this would include constraints such as the minimum separation between rovers, or the maximum slope traversable by a rover.

When comparing the values of metrics computed for different rovers or the same rover over multiple duty periods, it is important to establish a basis of comparison that identifies which of these performance dimensions predominate and how these dimensions combine to establish performance expectations. Considerations include changes to or differences between the rover equipment, features of the environment that significantly affect rover operations, and changes in safety constraints.

\section{Acknowledgments}

We thank Hans Utz, Tod Milam, David Lees, and Matt Deans for assisting with the development and implementation of real-time robot performance monitoring.

This work was partially supported by the NASA Astrobiology Science and Technology Instrument Development program, the NASA Exploration Systems Technology Development Program under the "Human-Robotic Systems" project and the NASA Small Business Innovative Research (SBIR) program.

\section{References}

1. Garcia E, Gonzalez de Santos P (2004) Mobile-robot navigation with complete coverage of unstructured environments. Robot Auton Syst 46:195-204

2. Wong SC, Middleton L, MacDonald BA (2002) Performance metrics for robot coverage tasks. In: Proc Australasian Conf Robot \& Autom, Auckland, New Zealand, 7-12

3. Mei Y, Lu Y-H, Hu YC, Lee CSG (2006) Deployment of mobile robots with energy and timing constraints. IEEE Trans. Robot 22 3:507-522

4. Fong T, Bualat M, Edwards L, Fluckiger L et al (2006) Human-robot site survey and sampling for space exploration. In: Proc AIAA Space Conf, San Jose, CA

5. Carpin S, Pillonetto G (2003) Robot motion planning using adaptive random walks. In: Proc IEEE Intl Conf Robot \& Autom, Taipei, Taiwan, 3809-3814 
6. Nakamura Y, Sekiguchi A (2001) The chaotic mobile robot. IEEE Trans. Robot \& Autom 17 6:898-904

7. Tunstel E, Anderson G, Wilson E (2007) Autonomous mobile surveying for science rovers using in situ distributed remote sensing. In: Proc IEEE Intl Conf Syst, Man, Cybern, Montreal, 2348-2353

8. Anderson GT, Hashemi RR, Wilson E, Clark M (2000) Application of cooperative robots to search for water on Mars using distributed spectroscopy. In: Proc Intl Symp Robot \& Appl WAC, Maui, HI

9. Schmitt HH, Kulcinski GL, Sviatoslavsky IN, Carrier III WD (1992) Spiral mining for lunar volatiles. In: Proc 3rd Intl Conf Eng, Constr \& Oper in Space III, Denver, CO, 1162-1170

10. Fong T, Allen M, Bouyssounouse X, Bualat MG et al (2008) Robotic site survey at Haughton Crater. In: Proc 9th Intl Symp Artif Intell, Robot \& Autom in Space, Los Angeles, CA

11. Elfes A, Dolan J, Podnar G, Mau S, Bergerman M (2006) Safe and efficient robotic space exploration with tele-supervised autonomous robots. In: Proc AAAI Spring Symp, Stanford, CA, 104-113

12. Hashemi RR, Jin L, Anderson GT, Wilson E, Clark M (2001) A comparison of search patterns for cooperative robots operating in remote environments. In: Proc Intl Symp Inf Technol: Comput \& Coding, Las Vegas, NV, 668-672

13. Wilson EW, Tunstel EW, Anderson GT (2007) BioGAS spectrometer for biogenic gas detection and location on the surface of Mars. In: Proc AIAA Infotech@Aerosp Conf \& Exhib, Rohnert Park, CA

14. Hashmonay RA, Yost, MG (1999) Innovative approach for estimating fugitive gaseous fluxes using computed tomography and remote optical sensing techniques. J Air Waste Manag Assoc 49 8:966-972

15. Mei Y, Lu Y-H, Hu YC, Lee CSG (2004) Energy-efficient motion planning for mobile robots. In: Proc IEEE Intl Conf Robot \& Autom, New Orleans, LA, 4344-4349

16. Shillcutt KJ (2000) Solar based navigation for robotic explorers. Doctoral Thesis, Robotics Institute, Carnegie Mellon University, Pittsburgh, PA, CMU-RI-TR-00-25

17. Tunstel E (2007) Operational performance metrics for Mars Exploration Rovers. J Field Robot: Quant. Perf. Eval. of Robot \& Intell Syst 24 8-9:651-670

18. Sukhatme GS, Bekey GA (1996) Multicriteria evaluation of a planetary rover. In: Proc IEEE Intl Conf Robot \& Autom, Minneapolis, MN

19. Schreckenghost D, Fong T, Milam T (2008) Human supervision of robotic site surveys. In: Proc $6^{\text {th }}$ Conf Hum/Robot Technol \& Vis for Space Explor, STAIF, Albuquerque, NM

20. Schreckenghost D, Fong T, Milam T, Pacis E, Utz, H (2009) Real-time assessment of robot performance during remote exploration operations. In: Proc IEEE Aerosp Conf, Big Sky, MT

21. Gernhardt M (2005) Work efficiency indices. Presentation at NASA Johnson Space Center, Houston, TX

22. Arnold J (2006) Towards a framework for architecting heterogeneous teams of humans and robots for space exploration. MS Thesis, Dept Aeronaut \& Astronaut, Massachusetts Institute of Technology, Cambridge, MA

23. Fong T, Bualat M, Deans M et al (2008) Field testing of utility robots for lunar surface operations. In: Proc AIAA Space Conf, San Diego, CA, AIAA-2008-7886 